\title{
THE SIMPLEST INVOLUTORIAL TRANSFORMATION CONTAINED MULTIPLY IN A LINE COMPLEX*
}

\section{BY VIRGIL SNYDER}

Involutorial birational transformations contained multiply in a linear line complex are interesting, as they furnish the simplest examples of involutions that are probably irrational. The following example is the simplest possible case of such a transformation; it possesses features that are characteristic, and may itself be irrational. $\dagger$

Let $\left(0,0, z_{3}, z_{4}\right)$ be a variable point on the line $x_{1}=0, x_{2}=0$, and $\lambda_{1} x_{3}{ }^{2}+\lambda_{2} x_{4}{ }^{2}=0$ an involution $I_{2}$ of pairs of planes of a pencil, the axis being skew to the first line. Let us suppose that the point $(z)$ and the planes $(\lambda)$ are connected by the relation $z_{3} \lambda_{1}{ }^{2}+z_{4} \lambda_{2}^{2}=0$. A point $(y)$ of space determines the pair of planes $x_{3}^{2} y_{4}^{2}-x_{4}^{2} y_{3}^{2}=0$ of the pencil; hence $z_{3}=y_{3}^{4}, z_{4}=y_{4}^{4}$. A point $(x)$ on the line joining $(y)$ to $(z)$ has coordinates of the form $\rho x_{1}=\sigma y_{1}, \rho x_{2}=\sigma y_{2}, \rho x_{3}=\sigma y_{3}+\tau z_{3}, \rho x_{4}=\sigma y_{4}+\tau z_{4}$. This line meets the plane conjugate to that determined by $(y)$ in $\left(y^{\prime}\right)$, corresponding to $\sigma=y_{3}{ }^{3}+y_{4}{ }^{3}, \tau=-2$.

The points $(y),\left(y^{\prime}\right)$ are therefore associated in an involutorial birational transformation, the equations of which have the form

$$
\left\{\begin{array}{l}
\rho x_{1}^{\prime}=\left(x_{3}^{3}+x_{4}^{3}\right) x_{1}, \\
\rho x_{2}^{\prime}=\left(x_{3}^{3}+x_{4}^{3}\right) x_{2}, \\
\rho x_{3}^{\prime}=\left(x_{4}^{3}-x_{3}^{3}\right) x_{3}, \\
\rho x_{4}^{\prime}=\left(x_{3}^{3}-x_{4}^{3}\right) x_{4} .
\end{array}\right.
$$

The transformation $I_{4}$ is contained doubly in the special linear line complex, the axis of which is $x_{1}=0, x_{2}=0$ in the sense that each line of the complex contains two pairs of conjugate points in $I_{4}$. Every plane through the axis is transformed into

* Written under the auspices of the Heckscher Foundation for the Promotion of Research, established by Mr. August Heckscher at Cornell University. Presented to the Society, April 6, 1928.

$\dagger$ Another involution of order 2 that is probably irrational is described in the Giornale di Matematiche, vol. 61 (1923), pp. 125-128. 
itself, but the axis is not in the base of the web of surfaces conjugate to the planes of space.

In the correspondence $z_{4} y_{3}{ }^{4}-z_{3} y_{4}{ }^{4}=0$ between the points $(z)$ of the axis and the planes $(y)$ of the pencil through the fundamental line, there are five positions of the point such that a corresponding plane passes through it. Of these, two are the double planes of the quadratic involution $I_{2}$ which correspond to $z_{3}=0, z_{4}=0$. At these points all four associated planes coincide. The points in $y_{3}=0$ and in $y_{4}=0$ are paired in the harmonic homology having its center on the axis $x_{1}=0, x_{2}=0$ and its axis as the fundamental line. The two centers $(0,0,1,0)$ and $(0,0,0,1)$ are fixed points in $I_{4}$.

The other three points on the axis which contain an associated plane are $(0,0,1,1),(0,0, \omega, 1),\left(0,0, \omega^{2}, 1\right), \omega^{3}=1$. The respective planes of $I_{2}$ not passing through the points are $x_{3}+x_{4}=0$, $x_{3}+\omega x_{4}=0, x_{3}+\omega^{2} x_{4}=0$. The three points are isolated fundamental points of the first order, and their images are the corresponding planes. The relation between the elements at each of the fundamental points and the associated image plane is perspectivity. Every plane through the axis is transformed into itself.

The line $x_{3}=0, x_{4}=0$ is fundamental of order 3 . Any point $\left(y_{1}, y_{2}, 0,0\right)$ on it has for images the three lines joining the point to the three fundamental points on the axis. The locus of these lines is the triad of planes

$$
x_{3}-x_{4}=0, x_{3}-\omega x_{4}=0, x_{3}-\omega^{2} x_{4}=0 .
$$

Moreover, every point on the line $x_{3}=0, x_{4}=0$ is invariant. The pencil of planes through $x_{3}=0, x_{4}=0$ is invariant in $I_{4}$, but the individual planes are interchanged as in the quadratic involution $I_{2}$. The jacobian of the system consists of the six planes $x_{3}{ }^{6}-x_{4}{ }^{6}=0$ through the fundamental line, each counted twice.

The conjugate of an arbitrary line of the complex is a plane quartic curve $C_{4}$ lying in the plane determined by the line and the axis, meeting the given line in two pairs of conjugate points. These curves form a Jonquières net $\left|C_{4}\right|$, having a common triple point at the intersection of the plane with the fundamental line, with all three tangents common. The other three basis points are the three fundamental points on the axis. The 
Jonquières involution is not perspective. Each point of an arbitrary line of the plane, regarded as a point $(y)$, is associated with a point $(z)$ on the axis, and the point of intersection is not self-corresponding, hence the line $(y z)$ which also contains $\left(y^{\prime}\right)$ envelopes a conic.

But when $(y)$ describes a line skew to the axis, although each point $(y)$ uniquely determines a point $(z)$ on the axis, the same point $(z)$ is associated with four points $(y)$, hence the locus $(y z)$ is now a ruled surface of order 5, having (z) as a four-fold and the given line as simple directrix. This surface contains $C_{4}$, the conjugate of the given line, and the curve described by the other pair of points on each line $(y z)$. If $(a x)=0,(b x)=0$ define the given line, the image lies on the quadric

$$
\left(a_{1} x_{1}+a_{2} x_{2}\right)\left(b_{3} x_{3}-b_{4} x_{4}\right)-\left(b_{1} x_{1}+b_{2} x_{2}\right)\left(a_{3} x_{3}-a_{4} x_{4}\right)=0,
$$

which does not contain the given line unless $a_{3} b_{4}-a_{4} b_{3}=0$, that is, the line meets the axis. But in that case the quadric is composite,

$$
\left(b_{3} x_{3}-b_{4} x_{4}\right)\left(a_{1} x_{1}+a_{2} x_{2}+k b_{1} x_{1}+k b_{2} x_{2}\right)=0,
$$

and the image $C_{4}$ lies in the plane through the axis.

The conjugate of an arbitrary plane is a quartic ruled surface having the fundamental line for triple directrix. It also passes through the three fundamental points on the axis, and at each has for tangent planes those connecting these points with the fundamental line, hence two different surfaces, conjugates of two planes, touch each other along all three sheets through the fundamental line. The proper curve of intersection, of order 4 , meets the axis in the three fundamental points. The fundamental line counts for order 12 in the intersection of the two surfaces.

The conjugate surface contains the three lines joining the point of intersection of the given plane with the fundamental line to the three fundamental points on the axis. If a plane passes through a fundamental point on the axis, its conjugate is composite, having the associated fundamental plane as component. The linear line congruence having the axis and the fundamental line for directrices is invariant in $I_{4}$.

The involution $I_{4}$ is of genus 0 . The curve of intersection of any plane with its conjugate surface is composite, consisting 
of four concurrent lines. The plane $\sum a_{i} x_{i}=0$ is transformed into

$$
\left(x_{3}^{3}+x_{4}{ }^{3}\right)\left(a_{1} x_{1}+a_{2} x_{2}\right)+\left(x_{4}{ }^{3}-x_{3}{ }^{3}\right)\left(a_{3} x_{3}-a_{4} x_{4}\right)=0,
$$

hence the curve of intersection is defined by

$$
a_{3} x_{3}^{4}+a_{4} x_{4}^{4}=0, \quad \sum a_{i} x_{i}=0 .
$$

These four lines are arranged in two pairs, in perspective from the point in which the given plane meets the axis.

As the plane $\sum a_{i} x_{i}=0$ turns about any fixed line $\sum b_{i} x_{i}=0$ in it, this composite curve generates a quintic ruled surface $R_{5}$ having the fundamental line for four-fold directrix. If the Plücker coordinates of the given line are $\left(a_{j k}\right)$, the equation of the surface is

$a_{13} x_{1} x_{3}{ }^{4}+a_{23} x_{2} x_{3}{ }^{4}+a_{14} x_{1} x_{4}{ }^{4}+a_{24} x_{2} x_{4}{ }^{4}+a_{34} x_{3} x_{4}\left(x_{3}{ }^{3}-x_{4}{ }^{3}\right)=0$.

There is an $\infty^{4}$ system of such surfaces. Those through any point $(y)$ also contain $\left(y^{\prime}\right)$ and the other pair of conjugate points on the line $\left(y y^{\prime}\right)$. This $\infty^{3}$ subsystem is of the form

$$
\begin{aligned}
& A\left\{y_{3} y_{4}\left(y_{3}{ }^{3}-y_{4}{ }^{3}\right) x_{1} x_{3}{ }^{4}-y_{1} y_{3}{ }^{4} x_{3} x_{4}\left(x_{3}{ }^{3}-x_{4}{ }^{3}\right)\right\} \\
+ & B\left\{y_{3} y_{4}\left(y_{3}{ }^{3}-y_{4}{ }^{3}\right) x_{2} x_{3}{ }^{4}-y_{2} y_{3}{ }^{4} x_{3} x_{4}\left(x_{3}{ }^{3}-x_{4}{ }^{3}\right)\right\} \\
+ & C\left\{y_{3} y_{4}\left(y_{3}{ }^{3}-y_{4}{ }^{3}\right) x_{1} x_{4}{ }^{4}-y_{1} y_{4}{ }^{4} x_{3} x_{4}\left(x_{3}{ }^{3}-x_{4}{ }^{3}\right)\right\} \\
+ & D\left\{y_{3} y_{4}\left(y_{3}{ }^{3}-y_{4}{ }^{3}\right) x_{2} x_{4}{ }^{4}-y_{2} y_{4}{ }^{4} x_{3} x_{4}\left(x_{3}{ }^{3}-x_{4}{ }^{3}\right)\right\}=0 .
\end{aligned}
$$

If this system is mapped on the points of another space $S_{3}$ ' the representation establishes a $(1,4)$ correspondence between the points of the two spaces $S_{3}{ }^{\prime}, S_{3}$; all the points common to any triad of associated surfaces lie on a line, which belongs to the complex $p_{12}=0$. Moreover, all the surfaces pass through four fixed points on the line $\left(y y^{\prime}\right)$.

Consider the surfaces $T_{5}(r), T_{5}(s)$ of this system associated with two skew lines $r, s$, neither belonging to the complex $p_{12}=0$. Their residual intersection lies on the regulus defined by $r, s$ and the axis $k$. It is of type $(4,5)$ on the quadric containing the regulus. The two points in which the fundamental line $x_{3}=0, x_{4}=0$ meets the quadric $H_{2}(r, s, k)$ are four-fold points on $C_{9}$. A general $C_{9}$ of type $(4,5)$ has 16 apparent double points hence is of genus 12 . But this $C_{9}$ has two four-fold points with coplanar branches, hence it is of genus zero. 
The curves of this system which pass through the fixed points on the line $\left(y y^{\prime}\right)$ are the images of the straight lines of the space $S_{3}{ }^{\prime}$; all four image points in $S_{3}$ of a point of the line in $S_{3}{ }^{\prime}$ lie on the curve. The correspondence contains two coincidences. Each curve of the subsystem is invariant in $I_{4}$.

Since $C_{9}$ has three simple points on the axis, the images of which are three straight lines, and has two four-fold points on the fundamental line, the image of each of which is three straight lines, the residual is the curve $C_{9}$ of type $(4,5)$ having the same fundamental points; that is, $C_{9}$ is invariant in $I_{4}$.

If the system $\left|R_{5}\right|$ is mapped projectively on the hyperplanes of $S_{4}$ the result is a quadric variety, hence the double involution is rational, but this is not sufficient to conclude that $I_{4}$ is rational. If the more general system $\left|s_{1}+s_{4}\right|: l^{3} 3 P$ is mapped on the hyperplanes of an auxiliary space, much higher dimensions must be employed.

The product of $I_{4}$ by the central harmonic homology $(0,0,0,1)$, $x_{4}=0$, is a non-involutorial $T_{4}$, which leaves every line of a linear congruence invariant. The plane $x_{4}=0$ is composed of invariant points. Any ruled surface belonging to the congruence is invariant under an infinite discontinuous group of birational transformations. The simplest case of $k$ pairs is that of the form $\rho x_{1}{ }^{\prime}=\left(x_{3}{ }^{2 k-1}+x_{4}{ }^{2 k-1}\right) x_{1}$, etc. There are now $2 k-1$ fundamental points on the axis, each having the other plane of the pair in $I_{2}$ for conjugate. The fundamental line $x_{3}=0, x_{4}=0$ is of multiplicity $2 k-1$, its image surface consisting of a plane to each fundamental point on the axis.

A plane not containing the axis nor the fundamental line meets its conjugate surface in $k$ pairs of lines, all belonging to a pencil. When $k=1$, a known quadratic transformation results.

The product of the transformation $I_{2 k}$ and the axial harmonic homology $H: x_{1}{ }^{\prime}=x_{1}, x_{2}{ }^{\prime}=x_{2}, x_{3}{ }^{\prime}=-x_{3}, x_{4}{ }^{\prime}=-x_{4}$ is a new transformation $S=I_{2 k} H=H I_{2 k}$, which is contained to multiplicity $k-1$ in the same special linear complex, and has two planes $x_{3}=0, x_{4}=0$ of invariant points.

Cornell University 